\title{
Electric-Vehicle Power Converters Model-Based Design-for-Reliability
}

\author{
Alessandro Soldati, Giorgio Pietrini, Matteo Dalboni, and Carlo Concari
}

\begin{abstract}
Fully electric vehicles are rapidly gaining user and market interest worldwide, due to their zero direct emissions, appealing driving experience and fashionable perception. Unfortunately, cost, range and reliability have not reached the desired targets yet. Since consumers are prone to spend money to have a more reliable system, Design-for-Reliability will be a useful tool for the Design of tomorrow's EVs, justifying part of the increased cost for these products. In this work, a vertical model-based approach to design-for-Reliability of power converters for EVs is presented, paying special attention to thermally-induced aging. The design starts from various driving cycles, properly assembled to describe the vehicle mission, then load profiles for the converters are found and the resulting thermal stress is quantified. The converter lifetime can be estimated, taking into account also parameter dispersion, and requirements for the active thermal control of the parts modeled achieved, thus giving practical information to the system designers.
\end{abstract}

Index Terms - Design-for-Reliability, driving cycles, electric vehicles, model-based design, power converters.

\section{INTRODUCTION}

$\mathrm{E}$ LECTRIC Vehicles (EVs) and, especially, Fully Electric Vehicles (FEVs) are gaining more attention and market share every day. Things changed a lot with respect to the recent past: Internal Combustion Engines (ICEs) are getting difficult to design to fulfill pressing limitations from the particulates and carbon dioxide points of view. In the meantime, different car makers put many EV models on the market, dispelling the myth that mobility by electric energy is unfashionable [1]-[3].

Despite this positive trend for EVs, some issues still require close attention: the charging infrastructure needs to be widened, battery performance should be improved, the cost reduced and reliability increased [4], [5]. The last two points are somehow related: design and production costs are high because sales volume is still small, but also because creating affordable EVs requires a lot of research and manufacturing effort. Moreover, the electric part of a vehicle is still perceived as unreliable by many users, even by those that drive modern yet ICE-based vehicles.

In the continuous effort to reduce both cost and time-to-mar-

Manuscript received May 18, 2018. (Corresponding author: Alessandro Soldati.)

The authors are with the Power Electronics group of the Department of Engineering and Architecture, University of Parma, 43124 Parma, Italy (e-mail: alessandro.soldati@unipr.it; giorgio.pietrini@unipr.it; matteo. dalboni@studenti.unipr.it; carlo.concari@unipr.it).

Digital Object Identifier 10.24295/CPSSTPEA.2018.00010 ket, as well as to increase safety, the automotive industry introduced massively model-based design into its project workflow [6]-[8]. Certainly, this methodology can be applied to meet all the performance metrics of a vehicle as well as to improve the design of the implementation platforms which are in charge to host the control algorithms [6]. Another advantage deriving from the model-based design consists in the possibility to lead also system-level analysis, hence increasing the integration of different physical domains which unavoidably cohabit in complex systems [7]. Moreover, such approach can be leveraged to obtain a quantitative assessment of the health and reliability of complex and distributed systems [8]. It is thus obvious that model-based design is widely accepted by car manufacturers, and that new technologies should be developed according to this paradigm. Therefore, this work, which settles in the automotive scenario, can be regarded as an effort to provide a novel design strategy of power converters from a vehicle-system-level point of view.

When reliability of modern power converters is concerned, the approach described by Design-for-Reliability (DfR), rather than reliability testing, is the preferred choice, as it has already been proposed for photovoltaics (PV) and energy systems based on renewables [9]-[11]. Indeed, for these applications life-time prediction is very important because the cost of renewable energy and hence its greater convenience over fossil energy depends directly on the number of faults and unexpected maintenance interventions which are required for the proper operation of $\mathrm{PV}$ and wind plants. Thus, in this work DfR is borrowed from the renewable energy field, where it represents a consolidated methodology, and it is exploited as a means to make automotive power converters more reliable and, consequently, to make EVs more desirable for consumers.

Furthermore, it is worth observing that DfR implies a paradigm switch towards the so called Physics of Failure (PoF) since this approach implies the detection of the root-causes of failure as stated in [9]-[11].

In the DfR method the designer effort is concerned with reliability from the early stages of the project. Methodologies that account for time dependent parameters and varying operating conditions are introduced, besides the more traditional, statistics-based approach.

This work proposes a possible workflow to design reliable power converters to be used in EVs, accounting also for design parameters of the vehicle that do not strictly belong to the inverter. To this aim, simulations of one year of realistic vehicle use are performed in order to determine the junction temperature profile of the power devices given the year-long 
mission profile. From the obtained temperature profile, rainflow counting and reliability models are used to finally determine the expected lifetime of the power converters. The simulation includes a set of several mechanical, electrical, and thermal parameters. The number and type of parameters used, as well as several assumptions, have been chosen in order to strike an optimal compromise between accuracy and simulation time. The parameter values have been determined from various sources, including scientific literature (for vehicle mechanical parameters) and device data sheets (for power electronic devices).

This work aims to satisfy both the manufacturer side, providing a completely model-based flow, and the user side, taking advantage of DfR to effectively guarantee lifetime and reliability performances. Similar works in this field exist; with respect to [12], more details are experienced in this work, paying special attention to models and focusing less on data collection. Comparing this work to [4], here a major effort in the powertrain modeling is undertaken, including also e-motor parameters and control.

Several of the previous works on this matter rely on motor models with a very low detail and assume a linear electrical behavior in every operating point. In order to have an accurate evaluation of the motor current request for a specific operating point, different control techniques must be considered. The approach proposed in this paper, takes into account the two main working regions of the Interior Permanent Magnet (IPM) motors: the Constant Torque Speed Range (CTSR), subject to the maximum current limit only, and the Constant Power Speed Range (CPSR), where also the maximum voltage limits have to be met.

The fitting parameters used in the reliability model (i.e. Coffin-Manson) are unavoidably affected by uncertainty, that is usually dealt with by means of Montecarlo analysis [9], [10], [11], [13], [14]. The authors of this paper present a Montecarlo analysis of the final results to analyze the effects of reliability model parameter dispersion.

All the above consider thermally induced material fatigue as the main failure mechanism; the battery, that is indeed a key part for vehicle system reliability, is neglected for now, since the focus is on power converter design. Future work should address this weakness.

\section{System ReQuiremENTS}

\section{A. Functional Requirements}

The realization of EVs, despite being quite complex, is rooted in very simple functional requirements: the vehicle should be able to carry its passengers wherever they want, in a comfortable and inexpensive way, while assuring safety into disparate traffic and environmental conditions. The main difficulty is the determination of a clear and quantitative definition of those aspects. Comfort, environment and traffic explicit models are outside the scope of this work, but "carrying passengers to their destination" can be accounted for by means of driving cycles (DCs). DCs come from the effort to substitute complex and
TABLE I

Physics Parameters Used into Model Equations

\begin{tabular}{ccc}
\hline \hline Symbol & Description & Unit \\
\hline$\Delta t$ & Simulation time step & $\mathrm{s}$ \\
$\rho$ & Air density & $\mathrm{kg} / \mathrm{m}^{3}$ \\
$g$ & Gravity acceleration & $\mathrm{m} / \mathrm{s}^{2}$ \\
\hline \hline
\end{tabular}

random processes with repeatable and deterministic quantities, without losing the statistical properties of the former [15], [16]. From the lifetime point of view, a single driving cycle is supposed to have little impact on the overall system lifespan, since durations are usually limited to half an hour; moreover, they are often not so meaningful for the real behavior of a vehicle [4]. If "standard" driving cycles are used, it is worth assuming a specific distribution of different driving cycles on a longer period. This allows to gather more variability than that available in a single, specific driving cycle, and to account for day-to-day aging of the vehicle system.

\section{B. Reliability Requirements}

Reliability requirements for EVs are the same of traditional ICE cars: to achieve a wide adoption of the new form of mobility, users should have the possibility to buy a new EV while, at least, maintaining the same expected lifetime. This supports in amortizing the purchase expense, that is still higher for EVs with respect to ICE vehicles. However, it is worth pointing out that the operating costs of owning an electric vehicle can be lower than those connected to other types of vehicle [3].

Roughly, for a typical consumer, the ownership of a vehicle ranges from 10 to 15 years [2], [3]: during this period, the vehicle is expected to be healthy, safe and reliable. As a matter of fact, in the United States vehicle and powertrain manufacturers must grant a 10 years warranty, which is an apparent indication of the minimum life expectation of a generic light-duty vehicle. Consequently, also EVs are required to meet at least a comparable lifespan.

\section{System Modeling}

The vehicle is a complex system; hence its modeling can be a difficult task. Various degrees of detail can be retained or abandoned, depending on the desired target. In the following, the vehicle is described by four models. The first is concerned with the mission profile and the dynamic constraints, the second describes the electrical machines and their power electronic converter, then a thermal model is used to infer the temperature of the relevant parts. Lastly, proper reliability models are introduced to complete the tool collection needed for DfR in EVs. The symbols used in the following models and their descriptions and units of measure are collected in TABLE I to TABLE V.

\section{A. Dynamic-Energetic Model}

The dynamic request coming from a driving cycle can be transformed into a power request by using the dynamic-energetic 
TABLE II

Vehicle Parameters Used into Model Equations

\begin{tabular}{ccc}
\hline \hline Symbol & Description & Unit \\
\hline$m$ & Vehicle mass & $\mathrm{kg}$ \\
$J_{w}$ & Wheel inertia & $\mathrm{kg} \mathrm{m}^{2}$ \\
$J_{m}$ & EM rotor inertia & $\mathrm{kg} \mathrm{m}^{2}$ \\
$r$ & Wheel radius & $\mathrm{m}$ \\
$S$ & Cross-sectional area of the vehicle & $\mathrm{m}^{2}$ \\
$C_{x}$ & Drag coefficient & - \\
$f_{o}$ & Friction coefficient, static & - \\
$k$ & Friction coefficient, motion & $\mathrm{s}^{2} / \mathrm{m}^{2}$ \\
$\tau$ & Reduction ratio motor-wheel & - \\
$n_{m}$ & Number of e-motors & - \\
\hline \hline
\end{tabular}

model presented here. It accounts for the inertia of both wheels and electric motors, the vehicle mass, aerodynamic and rolling friction. It supposes that the vehicle has $n_{m}$ electric motors, each coupled to the wheels by a fixed-ratio $(\tau)$ gearbox and, possibly, a differential gear. The differential is neglected from the energetic point of view, since it is assumed ideal. This is consistent with the driving cycles not providing information about the trajectory followed by the vehicle.

Under these assumptions, the overall friction force acting on the vehicle is ascribed to rolling and aerodynamics, represented by the first and second terms of (1), respectively. There, it can be seen that a square-law dependence on the vehicle speed $v$ exists.

$$
F_{\mu}=\left(f_{0}+k v^{2}\right) m g+\frac{1}{2} \rho C_{x} S v^{2}
$$

The power needed to change the vehicle speed in a certain amount of time depends on the frictional force that needs to be won $\left(F_{\mu}\right)$, the vehicle parameters and the magnitude of the speed change, as described by (2), which is obtained from the kinetic energy conservation.

$$
P_{r e q}=\left[\left(m+\frac{4 J_{w}}{r^{2}}+\frac{n_{m} \tau^{2} J_{m}}{r^{2}}\right) \frac{\mathrm{d} v}{\mathrm{~d} t}+F_{\mu}\right] v
$$

The power request will be satisfied by the traction system, possibly distributed on several $\left(n_{m}\right)$ motors; under the hypothesis of a straight-going vehicle, the torque $(T)$ and speed $(\omega)$ are almost equally distributed among them. Their effective value depends also on the fixed ratio $\tau$ of the gearbox, as stated by (3).

$$
\begin{aligned}
& T_{r e q}=\frac{\mathrm{P}_{r e q} r}{n_{m} \tau \nu} \\
& \omega_{r e q}=\frac{v p \tau}{r}
\end{aligned}
$$

Equations (1)-(3), when fed with a speed vs. time profile, i.e. a driving cycle, can thus yield the torque and electrical speed request for a single e-motor inside the powertrain, keeping into account many vehicle parameters.

\section{B. Electric Model}

The electric model is devoted to project the power request on motors and power converters. The model is considered an ideal transducer (without losses), while the non-ideal behavior of the converter (motor drive) is accounted for, to obtain the thermal stress that it undergoes during the operation of the vehicle. The electric model is thus split into two parts: the inverse motor model and the inverter loss model, presented below.

\section{1) Inverse Motor Model}

In this work, the e-motor is supposed to belong to the category of IPM synchronous machines, since this is the most common choice for EVs; the generated torque can approximately be expressed as in (4), where $\lambda_{m}, L_{d}$ and $L_{q}$ are considered constant over the entire working range (hypothesis of linearized motor).

$$
T=\frac{3}{2} p\left[\lambda_{m}+\left(L_{d}-L_{q}\right) i_{d}\right] i_{q}=\left(A+B i_{d}\right) i_{q}
$$

The second term is a simpler expression that will be used in the following to find the explicit control currents for each working point; the parameters are $A=3 p \lambda_{m} / 2$ and $B=3 p\left(L_{d}-L_{q}\right) / 2$. For the control currents to be determined, the relevant limits of the motor needs to be known: in this model, they are analytically determined from the motor and drive parameters themselves. The limiting values come from the maximum converter current modulus $\left(I_{\max }\right)$, the maximum phase voltage $V_{\max }$ (that descends from the DC link voltage $\left.V_{d c}, V_{\max }=V_{d c} / \sqrt{3}\right)$ and the maximum running speed $\omega_{\max }=\frac{2 \pi p}{60} \omega_{r p m, \max }$. The explicit limits of voltages and currents in rotating frame coordinates, with amplitude-conserving Clarke-Park transform, are expressed in (5).

$$
\begin{aligned}
& \left|i_{d q}\right|=\left|i_{d}+j i_{q}\right|=i_{d}^{2}+i_{q}^{2}<I_{\max }^{2} \\
& \left|v_{d q}\right|=\left|v_{d}+j v_{q}\right|=v_{d}^{2}+v_{q}^{2}<V_{\max }^{2}
\end{aligned}
$$

Firstly, starting from $I_{\max }$, the $d q$-currents for the maximum torque can be determined as in (6):

$$
\begin{aligned}
& i_{d, T \max }=\frac{-A+\sqrt{A^{2}+8 B^{2} I_{\text {max }}^{2}}}{4 B} \\
& i_{q, T \max }=\sqrt{I_{\text {max }}^{2}-i_{d, \text { max }}^{2}}
\end{aligned}
$$

so that the maximum torque can be easily computed as $T_{\max }=\left(A+B_{i d, T \max }\right) i_{q, T \max }$. Once the maximum torque is known, the base speed can be determined as in (7). This quantity is not critical for the control, but it is a useful value for debugging the model.

$$
\omega_{\text {base }}=\frac{v_{\max }}{\sqrt{\left(\lambda_{m}+L_{d} i_{d, T \max }\right)^{2}+\left(L_{q} i_{q, T \max }\right)^{2}}}
$$


TABLE III

Motor Parameters Used into Model Equations

\begin{tabular}{ccc}
\hline \hline Symbol & Description & Unit \\
\hline$p$ & Number of pole pairs & - \\
$L_{d}$ & D-axis inductance & $\mathrm{H}$ \\
$L_{q}$ & Q-axis inductance & $\mathrm{H}$ \\
$\lambda_{m}$ & PM linked flux & $\mathrm{V} \mathrm{s}$ \\
$I_{\max }$ & Maximum current & $\mathrm{A}$ \\
$V_{d c}$ & DC link voltage & $\mathrm{V}$ \\
$\omega_{r p m, \max }$ & Maximum mechanical speed & $\mathrm{rpm}$ \\
\hline \hline
\end{tabular}

When the torque request is below the $T_{\max }$ limit, a good control algorithm is represented by the MTPA (Maximum Torque Per Ampere) trajectory in the $i_{d q}$ plane. This locus is obtained by equating (4) to the requested torque $T_{\text {req }}$, while imposing the minimum current modulus (current circle tangent to iso-torque curve). The $i_{q}$ current can be obtained using (8):

$$
i_{q}=\frac{T}{A+B i_{d}}
$$

This results in a fourth-order polynomial (9); out of the general four solutions, the one of interest is the only real and negative one.

$$
B^{3} i_{d}^{4}+3 A B^{2} i_{d}^{3}+3 A^{2} B i_{d}^{2}+A^{3} i_{d}-B T_{r e q}^{2}=0
$$

This approach allows to find the control point necessary to achieve any torque below the maximum one, with speed below the base value (CTSR operating region). It is possible to determine the operating voltage of the motor, recalling (10):

$$
v_{d q}=v_{d}+j v_{q}=-\omega L_{q} i_{q}+j \omega\left(L_{d} i_{d}+\lambda_{m}\right)
$$

If the threshold of (5) is exceeded, the MTPA trajectory cannot be used any longer, and the control point should be determined by the flux-weakening technique intersecting the current circle with the voltage ellipsis in the $d q$ plane (CPSR operating region). This gives the polynomial (11), again with order four:

$$
\begin{aligned}
& B^{2} L_{d}^{2} i_{d}^{4}+\left(2 A B L_{d}^{2}+2 \lambda_{m} L_{d} B^{2}\right) i_{d}^{3}+ \\
& \left(B^{2} \lambda_{m}^{2}+4 A B \lambda_{m} L_{d}+A^{2} L_{d}^{2}-B^{2} V_{\max }^{2} / \omega^{2}\right) i_{d}^{2}+ \\
& \left(2 A B \lambda_{m}^{2}+2 A^{2} \lambda_{m} L_{d}-2 A B V_{\max }^{2} / \omega^{2}\right) i_{d}+ \\
& \left(A^{2} \lambda_{m}^{2}+L_{q}^{2} T_{r e q}^{2}-V_{\max }^{2} A^{2} / \omega^{2}\right)=0
\end{aligned}
$$

In this case the desired solution is the maximum among the negative (and real) ones. If all those constraints lead to unacceptable solutions, it means that the requested operating point is unachievable by the motor. Detailed description and units of measure of the parameters of this model are reported in TABLE III.

\section{2) Inverter Loss Model}

The inverse motor model described in Section III-B-1) transforms the mechanical requests coming from the vehicle into electric requests that need to be fulfilled by the motor converter.
TABLE IV

InVerter Parameters Used into Model Equations

\begin{tabular}{ccc}
\hline \hline Symbol & Description & Unit \\
\hline$V_{d c}$ & DC-link voltage & $\mathrm{V}$ \\
$f_{s w}$ & Switching frequency & $\mathrm{Hz}$ \\
$V_{c}$ & Switch voltage drop $(I>0)$ & $\mathrm{V}$ \\
$R_{c}$ & Switch on-state resistance $(I>0)$ & $\Omega$ \\
$V_{d}$ & Switch voltage drop $(I<0)$ & $\mathrm{V}$ \\
$R_{d}$ & Switch on-state resistance $(I<0)$ & $\Omega$ \\
$E_{\text {on }}$ & Turn-on energy & $\mathrm{J}$ \\
$I_{\text {Eon }}$ & Current of $E_{\text {on }}$ measure & $\mathrm{A}$ \\
$E_{\text {off }}$ & Turn-off energy & $\mathrm{J}$ \\
$I_{\text {Eoff }}$ & Current of $E_{\text {off }}$ measure & $\mathrm{A}$ \\
\hline \hline
\end{tabular}

Those, in turn, determine the inverter losses, that are the main cause of thermal cycling of the converter itself, thus leading to material fatigue.

Inverter losses in a traditional three-phase bridge architecture for battery-supplied vehicles can be ascribed mainly to the power switches, since no large magnetic components are involved. Device losses are determined by the inverter generated waveform, the phase-shift between voltage and current (load angle) and the voltage and current moduli, as obtained from the inverse motor model. The conduction loss is described analytically by (12):

$$
\begin{aligned}
P_{o n}= & \frac{I_{0}^{2}}{8}\left(R_{c}+R_{d}\right)+\frac{I_{0}}{2 \pi}\left(V_{c}-V_{d}\right)+ \\
& \frac{2 I_{0}^{2} V_{0}}{3 \pi V_{d c}}\left(R_{c}-R_{d}\right) \cos \varphi+\frac{I_{0} v_{0}}{4 V_{d c}}\left(V_{c}+V_{d}\right) \cos \varphi
\end{aligned}
$$

To achieve this very concise formulation, some hypotheses were needed. Firstly, the output waveform is supposed to be a PWM-generated sinusoid, where the third-harmonic injection is neglected for simplicity. Then, each device is modeled, in the on-state, by a linear relationship (with offset) between current and voltage; four independent values are needed to properly describe the switch under positive and negative currents, since conduction losses are usually different. These values, represented by $V_{c}, R_{c}, V_{d}$ and $R_{d}$ in TABLE IV can describe effectively both MOSFETs and IGBTs behavior. Since $V_{d}$ is the switch voltage when it carries negative current, it has a negative value, while the resistance $R_{c}$ is positive. For MOSFETs, typically, $V_{c}=V_{d}=0$ because they do not exhibit any threshold behavior, but $R_{c}=R_{d}>0$, because their behavior with respect to current is almost symmetrical. From this it descends that the conduction loss of a MOSFET-based converter is flat and independent of the load angle, if the current conduction is symmetric in the device itself.

Equation (12) was obtained averaging, on an output sinusoid period, the mean power loss on each PWM cycle, while accounting for the current direction, that is determined by the load (motor) angle $\varphi$.

A similar averaging approach was used to determine the switching losses over a sinusoidal period. In this case a loose dependence exists on the voltage, since each commutation happens with the entire DC link voltage $V_{d c}$, here assumed constant. 
However, during the sinusoid generation, the current changes, and as many switching events as the ratio between the switching and the fundamental frequency $\left(f_{s w} / f_{0}\right)$ happen. This suggests that averaging translates into a finite sum of energy packets; this computation was done by approximating the finite sum with a definite integral, which holds approximately for $f_{s w} / f_{0}>10$. The final result is described in (13), where the datasheet values $E_{\text {on }}$ and $E_{\text {off }}$ are scaled by their characteristic current.

$$
P_{s w}=\frac{2 f_{s w} I_{0}}{\pi}\left(\frac{E_{o n}}{I_{E o n}}+\frac{E_{o f f}}{I_{E o f f}}\right)
$$

It is shown that, at least under the given approximations, the switching loss depends only on the motor current and the switching frequency, but neither on the fundamental frequency nor on the load angle: this dependence is canceled by averaging over the output waveform. The parameters used in the model are described in TABLE IV.

\section{Thermal Model}

The thermal model represents the cooling system and its effects on the temperature of the parts of interest. This sub-system has a serious impact on the overall system reliability, at least for the thermally-induced faults. Four relevant points were chosen, out of which only three are non-trivial, so a three-time-constants network was initially devised. The "ambient" node (A) is kept at a constant temperature, while the other nodes are connected to each other through a simple Cauer thermal network. The non-trivial nodes are: junction (J), which is also where the power loss source is located, case (C), i.e. the device case, and the heatsink (S), that is the main source of cooling in the system.

Given the three time constants, a third-order ODE (Ordinary Differential Equation) is expected; an analytical solution is possible even in this case, but possibly complex. Moreover, the model is intended to be used to simulate an entire year of operation of a vehicle, which is quite a long period. Nonetheless, almost all driving cycles available have time base greater or equal than one second. With typical devices, the time-dependent effects of the junction and case capacitances are almost unnoticeable at this bandwidth. The system can thus be simplified, retaining all the thermal resistances and the heatsink capacitance $C_{s}$ only.

The result is a simple first-order ODE, that can be straightforwardly solved for the heatsink temperature as in (14), where $P_{\text {loss }}$ is the device power loss and $T_{s, 0}$ the temperature at the beginning of the sampling period (which coincides with the narrowest driving cycle time base):

$$
\begin{aligned}
T s= & \left(R_{s a} P_{\text {loss }}+T_{a}\right)\left[1-\exp \left(-\frac{\Delta t}{R_{s a} C_{s}}\right)\right]+ \\
& T_{s, 0} \exp \left(-\frac{\Delta t}{R_{s a} C_{s}}\right)
\end{aligned}
$$

Neglecting both $C_{j}$ and $C_{c}$, the case and junction temperatures are determined using only the resistance among those layers, re-
TABLE V

Thermal Parameters Used into Model Equations

\begin{tabular}{ccc}
\hline \hline Symbol & Description & Unit \\
\hline$R_{j c}$ & Junction-case thermal resistance & $\mathrm{K} / \mathrm{W}$ \\
$R_{c s}$ & Case-sink thermal resistance & $\mathrm{K} / \mathrm{W}$ \\
$R_{s a}$ & Sink-ambient thermal resistance & $\mathrm{K} / \mathrm{W}$ \\
$C_{j}$ & Junction thermal capacitance & $\mathrm{J} / \mathrm{K}$ \\
$C_{c}$ & Case thermal capacitance & $\mathrm{J} / \mathrm{K}$ \\
$C_{s}$ & Heatsink thermal capacitance & $\mathrm{J} / \mathrm{K}$ \\
$T_{a}$ & Ambient temperature & ${ }^{\circ} \mathrm{C}$ \\
\hline \hline
\end{tabular}

sulting in $T_{c}=T_{s}+R_{c s} P_{\text {loss }}$ and $T_{j}=T_{c}+R_{j c} P_{\text {loss }}$. The parameters are detailed in TABLE V.

\section{Reliability Model}

The reliability model is usually constituted by a collection of models, depending on the failure mechanisms and failing parts that are considered. These are collected from literature and computed in parallel, in order to see which is the first mechanism that determines the system failure. This technique is coherent with the PoF approach that is nowadays preferred to the older "testing for reliability" [14].

Before applying the reliability models, out of which we selected only that describing the thermal fatigue mechanism, the thermal loading of the device inside the converter should be analyzed. This can be accomplished by sound counting algorithms, among which the rainflow counting is the most renowned.

\section{1) Rainflow Counting}

Counting algorithms are used to infer the relevant number of fatigue cycles from a general mission profile. The rainflow counting used here is based on the standard [17], as implemented in the MATLAB environment. This implementation follows the three-point algorithm, but can work on a stream of data, without any specific need for sample reordering.

\section{2) Lifetime Models for the Power Devices}

When it comes to thermal fatigue in power electronics, the Norris-Landzberg model, as a modification of the basic Coffin-Manson equation to include frequency, is the flagship relationship. Despite more advanced models being available [18], the highest hurdle is represented by finding the proper parameters, that allow a precise representation of the device statistical behavior.

For this work, the expected number of cycles to failure $N_{f}$ of a specific cycling, with average junction temperature $T_{j, \text { avg }}$ and range $\Delta T_{j}$, is obtained by (15), which is the simple Coffin-Manson model:

$$
N_{f}=C \cdot \Delta T_{j}^{a} \cdot \exp \left(\frac{E_{a}}{k_{B} T_{j, \text { avg }}}\right)
$$

where $k_{B}$ is the Boltzmann constant and $C, a$ and $E_{a}$ are the reliability parameters, which were obtained from [4]. To take into account the distribution of the various cycles, as resulting 
TABLE VI

Parameters Used in the Simulation Presented as Example of the Design Workflow

\begin{tabular}{l|l|l|l}
\hline \hline$\Delta t=1 \mathrm{~s}$ & $k=1 \times 10^{-4} \mathrm{~s}^{2} / \mathrm{m}^{2}$ & $V_{c}=0 \mathrm{~V}$ & $R_{s a}=0.30 \mathrm{~K} / \mathrm{W}$ \\
$\rho=1.293 \mathrm{~kg} / \mathrm{m}^{3}$ & $R_{c}=4 \mathrm{~m}$ & $C_{j}=0 \mathrm{~J} / \mathrm{K}$ \\
$g=9.81 \mathrm{~m} / \mathrm{s}^{2}$ & $V_{d}=0 \mathrm{~V}$ & $C_{c}=0 \mathrm{~J} / \mathrm{K}$ \\
$m=1500 \mathrm{~kg}$ & $n_{m}=1$ & $R_{d}=4 \mathrm{~m}$ & $C_{s}=2000 \mathrm{~J} / \mathrm{K}$ \\
$J_{w}=250 \times 10^{-3} \mathrm{~kg} \mathrm{~m}^{2}$ & $p=4$ & $E_{\text {on }}=5.6 \mathrm{~mJ}$ & $T_{a}=20^{\circ} \mathrm{C}$ \\
$J_{m}=15 \times 10^{-3} \mathrm{~kg} \mathrm{~m}^{2}$ & $L_{d}=300 \mathrm{H}$ & $I_{\text {Eon }}=300 \mathrm{~A}$ & $=302500 \mathrm{~K}^{-\alpha}$ \\
$r=180 \times 10^{-3} \mathrm{~m}$ & $L_{q}=800 \mathrm{H}$ & $E_{\text {off }}=3.7 \mathrm{~mJ}$ & $a=-5.039$ \\
$S=2 \mathrm{~m}^{2}$ & $\lambda_{m}=85 \times 10^{-3} \mathrm{~V} \mathrm{~s}$ & $I_{\text {Eoff }}=300 \mathrm{~A}$ & $E_{a}=9.89 \times 10^{-20} \mathrm{~J}$ \\
$C_{x}=0.3$ & $I_{\text {max }}=400 \mathrm{~A}$ & $R_{\text {jc }}=0.10 \mathrm{~K} / \mathrm{W}$ & \\
$f_{0}=10 \times 10^{-3} \mathrm{~Hz}$ & $V_{d c}=650 \mathrm{~V}$ & $R_{c s}=0.17 \mathrm{~K} / \mathrm{W}$ & \\
\hline \hline
\end{tabular}

from the rainflow counting, the linear damage accumulation as described by Palmgren-Miner's rule was used [19]-[21].

\section{SimULATiON}

To obtain relevant information from the models described above, some secondary tasks need to be accomplished. They involve driving cycle preparation, transient simulation post-processing and, finally, result interpretation. These steps are detailed in the following, while the values for the parameters used are collected in TABLE VI.

\section{A. Preparation}

Driving cycle preparation is essential to obtain practically relevant results. Known cycles (ARTEMIS, EU and USA ones, ...) were collected and assembled in a long driving cycle (the master cycle), which can be regarded as a tangible element of novelty with respect to previous works. The construction procedure, in the effort to reproduce a plausible vehicle usage, accounts for relatively long stationary phases, with some driving moments interleaved. Fundamentally, the aforementioned extended driving cycle mirrors the endeavor to generate a significant virtual mission profile which is conceived to overcome the limitations of the single standard driving profiles. Indeed, as pointed out in [4], some driving cycles may lack of realism due to underestimate of maximum accelerations and, consequently they may mislead in damage assessment on power converters. Moreover, it is important to point out that rest phases in driving profiles have a paramount role for the sake of DfR of power electronics systems because they foster wide thermal cycles: these depict generally the major stressor for the electronic components [22].

The master cycle is based on three random variables: the drive/no-drive flag $\left(P_{d}\right)$, the driving frame index $\left(I_{d}\right)$ and the stop duration $\left(L_{s}\right) . P_{d}$ is a Bernoulli random variable, that determines, at each draw, if the vehicle will move or not in the following driving cycle. The driving probability was set to $10 \%$, to describe roughly a 2 hour/day use of the vehicle (approximately $35 \cdot 10^{3} \mathrm{~km} /$ year), in accordance with statistics asserting that, on average, a vehicle is idling more than $90 \%$ of the time [22]. If the vehicle moves, the $I_{d}$ random variable, with discrete uniform distribution, chooses one out of the available conventional driving cycles. If the vehicle rests, the $L_{s}$ variable, with uniform dis-

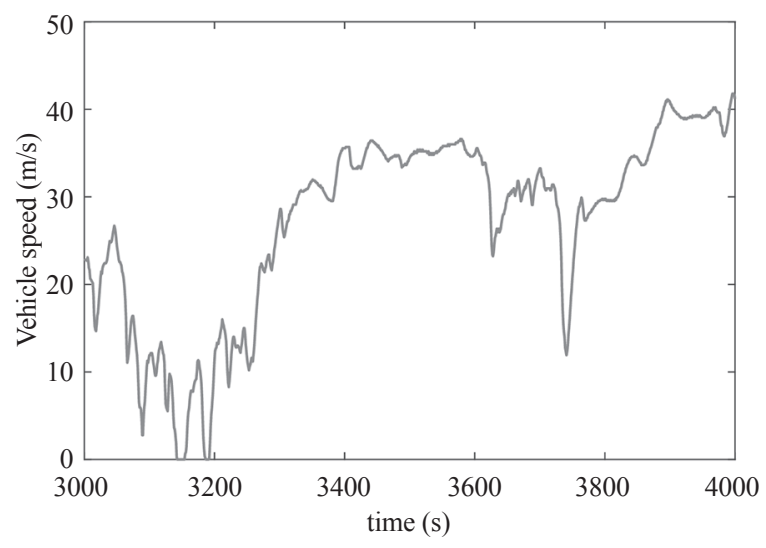

Fig. 1. Snapshot of a part of the driving cycle.

tribution between 0 and 1 , determines the rest duration in hours. At the moment, no special means to avoid driving by night is enforced, nor any feedback on the effective distance traveled.

\section{B. Post-Processing}

The transient simulation, running the models of section III, determines the junction temperature profile related to the master driving cycle, together with other information, such as the heatsink temperature, the dynamic request to the motor and the electrical quantities.

After that, the junction temperature profile is analyzed by rainflow counting and the consumed lifespan for a specific set of reliability parameters was determined. Since these parameters are usually affected by a large uncertainty, a Montecarlo analysis was carried out. Hence, each parameter was changed based on a Gaussian distribution with unitary mean and variable standard deviation, and the new lifespan determined, always using the same temperature profile obtained from the simulation. This results in a specific lifetime histogram, that can be fitted to various distributions to find detailed lifetime metrics.

\section{Results}

The transient simulation is capable of giving many quantities of interest. Some of them are reported in Fig. 1-Fig. 5. The driving cycle (a short snapshot) is represented in Fig. 1, with the resulting mechanical power request, to be satisfied by the e-motors 


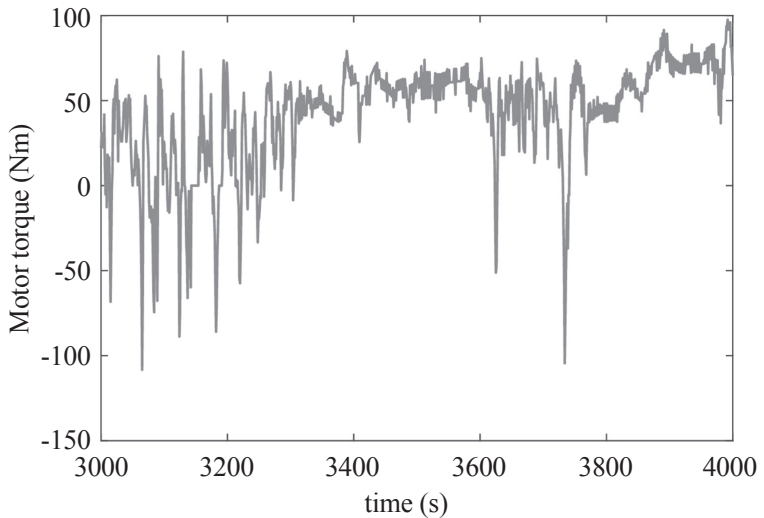

Fig. 2. Dynamic torque request corresponding to the driving cycle of Fig. 1.

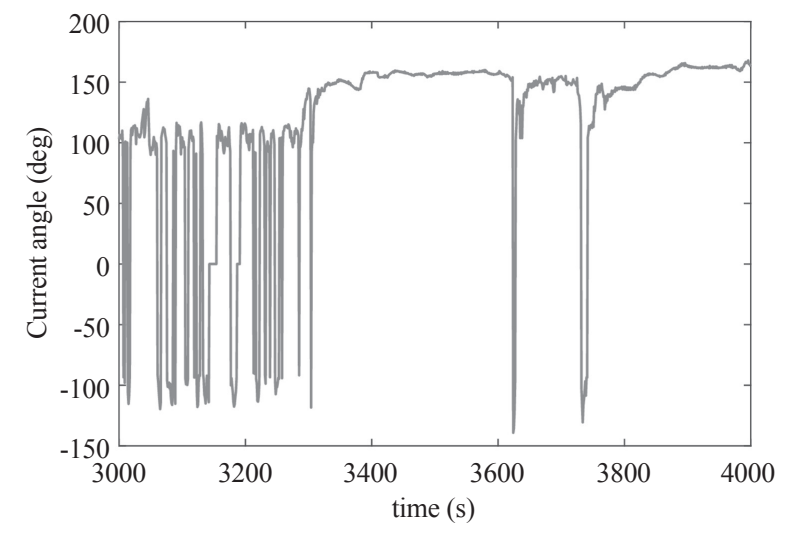

Fig. 3. Motor current angle in $d q$ axes.

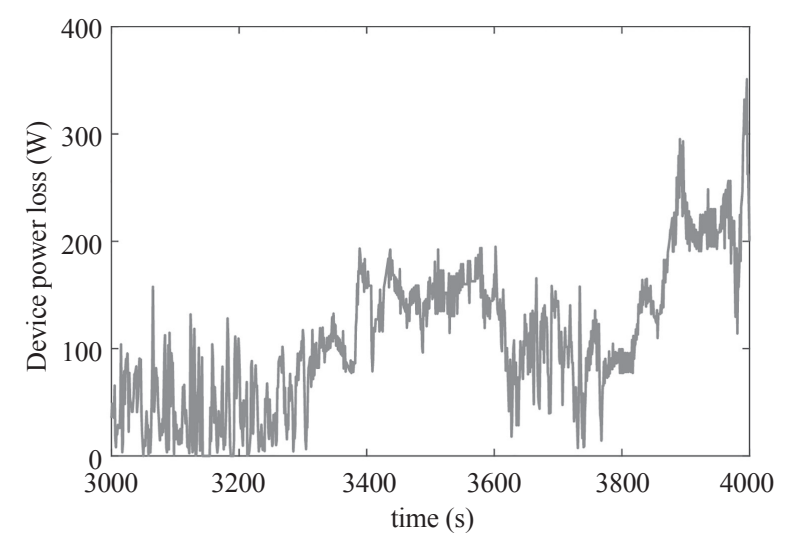

Fig. 4. Power loss on one inverter device, corresponding to the load profile of Fig. 3.

in the powertrain, in Fig. 2 . The $i_{d q}$ current angle, used to control the motor in a rotating reference frame, is depicted in Fig. 3; the power loss of a device in the inverter is in Fig. 4. Lastly, the device temperatures (junction, case and heatsink) are outlined in Fig. 5 stroked, dotted and dashed, respectively.

The results of the rainflow counting are reported in Fig. 6. It can be seen that a specific distribution of counts, coming from the master driving cycle, is obtained. Each count is used to apply Miner's rule and the fraction of consumed lifetime.

The aforementioned post-processing results in a distribu-

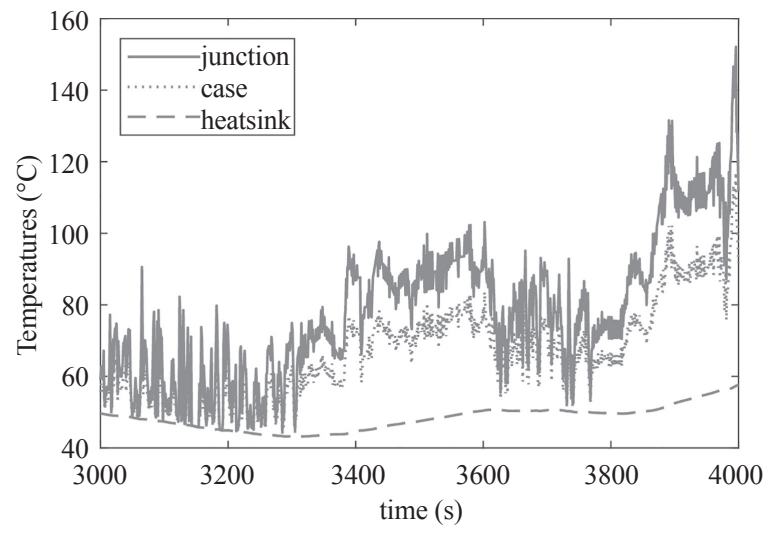

Fig. 5. Device temperatures due to the loss profile of Fig. 4.

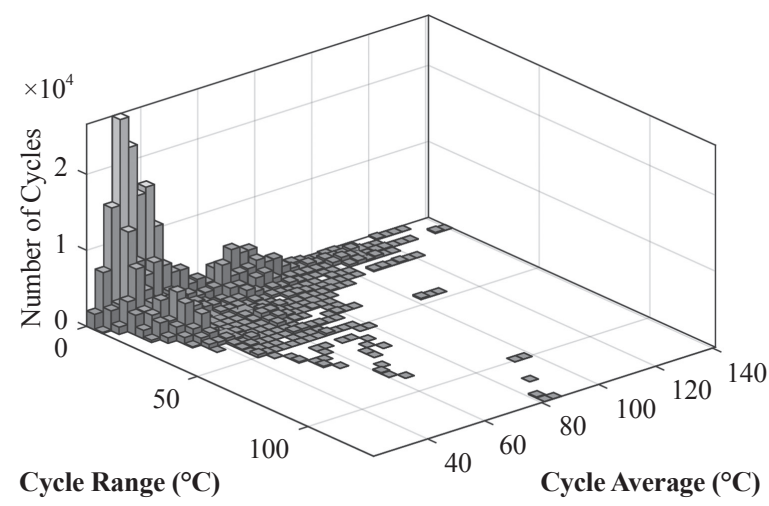

Fig. 6. Rainflow diagram of the junction temperature cycles under a oneyear-long driving cycle.

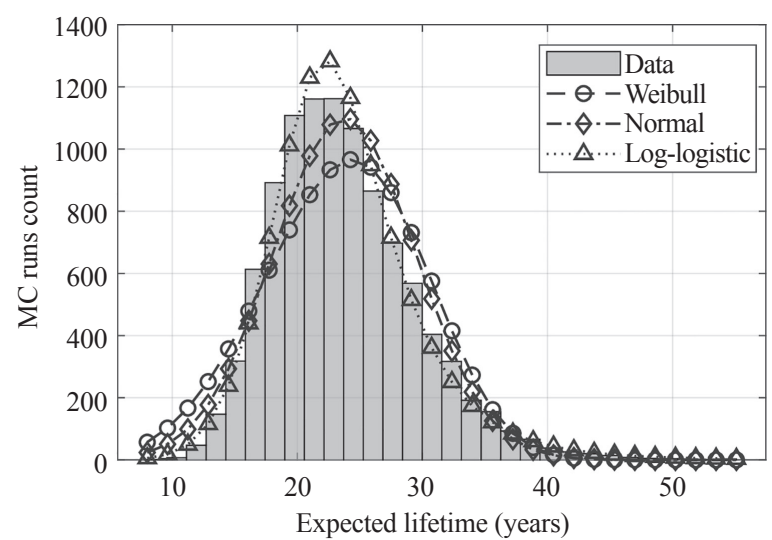

Fig. 7. Lifetime histogram from Monte Carlo simulations, with various fitting distributions.

tion described by the histogram of Fig. 7, where also Normal, Weibull and Log-logistic fitting distributions are represented. Fitting the Montecarlo data ( $10^{4}$ runs) to specific distributions allows to determine quantitatively the lifetime metrics, with statistical meaning. In TABLE VII the $B_{10}$ lifetime, i.e. the time after which $10 \%$ of the initial population has failed, is reported, as well as mean $(\mu)$ and standard deviation $(\sigma) . B_{10}$ can be sometimes more effective that a rough average value in describing the expected lifetime from a practical point of view. The distribution of Fig. 7 shows a prominent and fairly narrow peak; con- 
TABLE VII

Statistical Characterization of the Converter Lifetime, as Resulting From a One-Year-Long Master Driving Cycle and Monte Carlo Analysis All Quantities are Expressed in Years

\begin{tabular}{cccc}
\hline \hline Fitting & $B_{10}$ & $\mu$ & $\sigma$ \\
\hline Weibull & 15.2 & 23.6 & 6.3 \\
Normal & 16.5 & 23.8 & 5.7 \\
Log-logistic & 17.2 & 23.8 & 6.0 \\
\hline \hline
\end{tabular}

sequently, uncertainty on the lifetime model parameters does not affect significantly the lifetime estimate.

\section{CONCLUSION}

This paper describes a possible workflow to perform model-based Design-for-Reliability for power converters in EVs. Since it encompasses many vehicle parameters, it is able to correlate many design choices (also extraneous to the inverter itself) to the resulting lifetime change, in a statistical sense.

In the example reported here, a sufficient lifetime, also in the $B_{10}$ sense, was achieved, suggesting the correctness of the design choices.

Further work will deal with the sensitivity analysis of the lifetime to each parameter, while including also the battery properties and other aging mechanisms, to describe not only the effects on converter life, but also for the entire vehicle reliability.

\section{ACKNOWLEDGMENT}

The authors wish to thank Mr. Jos van der Geest for providing the catstruct MATLAB function, fundamental to support in driving cycle assembly.

\section{REFERENCES}

[1] G. Morrison, J. Stevens, and F. Joseck, "Relative economic competitiveness of light-duty battery electric and fuel cell electric vehicles," Transportation Research Part C: Emerging Technologies, vol. 87, pp. 183-196, 2018.

[2] W. Feng and M. Figliozzi, "An economic and technological analysis of the key factors affecting the competitiveness of electric commercial vehicles: A case study from the USA market," Transportation Research Part C: Emerging Technologies, vol. 26, pp. 135-145, 2013.

[3] P. Weldon, P. Morrissey, and M. O’Mahony, "Long-term cost of ownership comparative analysis between electric vehicles and internal combustion engine vehicles," Sustainable Cities and Society, vol. 39, pp. 578-591, 2018.

[4] N. Degrenne and S. Mollov, "Real-life vs. Standard driving cycles and implications on EV power electronic reliability," in IECON 2016 - 42nd Annual Conference of the IEEE Industrial Electronics Society, 2016, pp. 2177-2182.

[5] A. Barré, B. Deguilhem, S. Grolleau, M. Gérard, F. Suard, and D. Riu, "A review on lithium-ion battery ageing mechanisms and estimations for automotive applications," Journal of Power Sources, vol. 241, pp. 680-689, 2013.

[6] W. Chang, D. Roy, L. Zhang, and S. Chakraborty, "Model-based design of resource-efficient automotive control software," in 2016 IEEE/ ACM International Conference on Computer-Aided Design (ICCAD), 2016, pp. 1-8.

[7] J. Wan, A. Canedo, and M. A. A. Faruque, "Functional Model-Based Design Methodology for Automotive Cyber-Physical Systems," IEEE Systems Journal, vol. 11, no. 4, pp. 2028-2039, Dec. 2017.
[8] S. Reiter, M. Pressler, A. Viehl, O. Bringmann, and W. Rosenstiel, "Reliability assessment of safety-relevant automotive systems in a model-based design flow," in 2013 18th Asia and South Pacific Design Automation Conference (ASP-DAC), 2013, pp. 417-422.

[9] F. Blaabjerg, D. Zhou, A. Sangwongwanich, and H. Wang, "Design for reliability in renewable energy systems," in 2017 International Symposium on Power Electronics (Ee), 2017, pp. 1-6.

[10] H. Wang, F. Blaabjerg, K. Ma, and R. Wu, "Design for reliability in power electronics in renewable energy systems - status and future," in 4th International Conference on Power Engineering, Energy and Electrical Drives, 2013, pp. 1846-1851.

[11] Y. Yang, A. Sangwongwanich, and F. Blaabjerg, "Design for reliability of power electronics for grid-connected photovoltaic systems," CPSS Transactions on Power Electronics and Applications, vol. 1, no. 1, pp. 92-103, Dec. 2016.

[12] C. Geiger and G. Sarakakis, "Data driven design for reliability," in 2016 Annual Reliability and Maintainability Symposium (RAMS), 2016, pp. 1-6.

[13] H. Wang, K. Ma, and F. Blaabjerg, "Design for reliability of power electronic systems," in IECON 2012-38th Annual Conference on IEEE Industrial Electronics Society, 2012, pp. 33-44.

[14] H. Wang et al., "Transitioning to Physics-of-Failure as a Reliability Driver in Power Electronics," IEEE Journal of Emerging and Selected Topics in Power Electronics, vol. 2, no. 1, pp. 97-114, Mar. 2014.

[15] M. André, "The ARTEMIS European driving cycles for measuring car pollutant emissions," Science of The Total Environment, vols. 334335, pp. 73-84, 2004.

[16] T. Barlow, A Reference Book of Driving Cycles for Use in the Measurement of Road Vehicle Emissions: Version 3. IHS, 2009.

[17] ASTM-E1049-85(2017), Standard Practices for Cycle Counting in Fatigue Analysis. West Conshohocken, PA: ASTM International, 2017.

[18] R. Bayerer, T. Herrmann, T. Licht, J. Lutz, and M. Feller, "Model for Power Cycling lifetime of IGBT Modules-various factors influencing lifetime," in 5th International Conference on Integrated Power Electronics Systems, 2008, pp. 1-6.

[19] M. Miner, "Cumulative fatigue damage," Journal of applied mechanics, vol. 12, no. 3, pp. A159-A164, 1945.

[20] A. Palmgren, "Die Lebensdauer von Kugellagern (Durability of ball bearings)," $Z D V D$, vol. 68, p. 339, 1924.

[21] A. Czechowski and A. Lenk, "Miner's Rule in Mechanical Tests of Electronic Parts," IEEE Transactions on Reliability, vols. R-27, no. 3, pp. 183-190, Aug. 1978.

[22] D. Hirschmann, D. Tissen, S. Schröder, and R. W. D. Doncker, "Reliability prediction for inverters in hybrid electrical vehicles," in 2006 37th IEEE Power Electronics Specialists Conference, 2006, pp. 1-6.

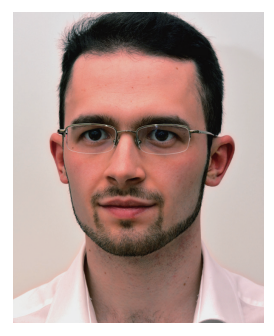

Alessandro Soldati got his $\mathrm{PhD}$ in Information Technology at the University of Parma in 2018, where he is currently a research assistant working on electric powertrains. His research interests focus on active gate drivers for wide band-gap devices, high-density power converters and control algorithms. He participates to different teaching activities and co-tutors theses on Power Electronics projects. Currently he is guest lecturer of the course "Embedded Systems Design for Industrial Electronics" at the University of Parma. He is also CTO of eDriveLAB, a spinoff company in vehicle electrification, and author of one patent.

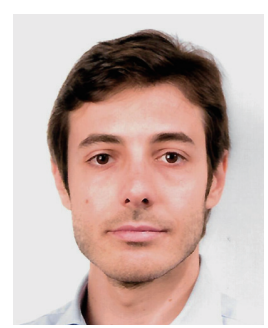

Giorgio Pietrini received the B.Sc. and M.Sc. degrees from the Department of Information Engineering at University of Parma, Italy, in 2009 and 2014 respectively. He is now pursuing the Ph.D. degree in Information Technology at the same university. His research interests include electrical machine modeling and design with special regard to permanent magnet synchronous motors for high performance automotive traction applications. 


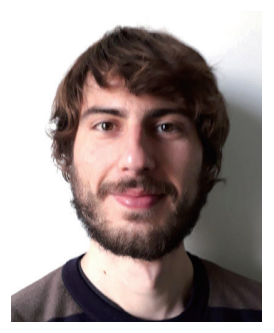

Matteo Dalboni received his Master degree in mechanical engineering from the University of Parma (Italy) in 2017. Currently, he is conducting the doctoral studies at the University of Parma; his research area concerns the electric vehicle dynamics and control. Principally, he is focusing on wheeled vehicle modeling techniques and on the development of control algorithms oriented to the torque allocation in fully electric vehicles with independent motors.

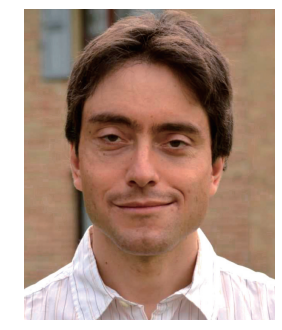

Carlo Concari received the Ph.D. degree in information technology from the University of Parma, Italy, in 2006. He is currently an Associate Professor with the Department of Engineering and Architecture, University of Parma. His research interests include power electronics, digital drive control, electric propulsion, static power converters, and electric machines diagnostics. He is the author or coauthor of more than 70 papers. 